\title{
Simulating human occupancy in an experimental laboratory setting
}

\author{
Joseph Martin Petersen ${ }^{1,2}$ \\ ${ }^{1}$ Washington State University, School of Electrical Engineering and Computer Science, Richland, Washington, USA \\ ${ }^{2}$ Pacific Northwest National Laboratory Energy Policy \& Economics, Richland, Washington. USA
}

\section{Email address:}

Joseph.m.petersen@gmail.com

\section{To cite this article:}

Joseph Martin Petersen. Simulating Human Occupancy in an Experimental Laboratory Setting. International Journal of Sustainable and Green Energy. Vol. 4, No. 1, 2015, pp. 7-10. doi: 10.11648/j.ijrse.20150401.12

\begin{abstract}
Energy conservation within a residential home is a primary focus for both home owners and power utilities throughout the country. Developing a technology to model, detect, and measure human occupancy would allow for laboratory settings to more accurately model residential energy use without the need for actual human activity within the space. An accurate way to measure occupancy is through detecting the latent and sensible heat that is generated by activities within the home. As industry facilities move forward with research, innovative ways to model every aspect of a residential home comes into play. These research settings require the development of technology that appropriately models and detects human activity within a residential home.
\end{abstract}

Keywords: Alternative, Energy, Modeling, Robotics

\section{Introduction}

Laboratory settings that model whole house energy use have struggled to find an accurate and efficient way to simulate human occupancy within these closed loop facilities. Both the latent and sensible heat, in reference to human generation, can account for large changes in the whole house energy use over time. Generating these representative loads can be effected by many external parameters. In order to acquire an accurate representation of the whole house energy use, a system is needed to model the day to day humidity generation of a typical family. This paper proposes the development of a standalone occupancy simulating system.

\section{Background}

Many laboratory studies have been conducted on the whole house energy use of a residential home with the hopes of understanding how differing variables will alter the total energy use. Energy efficient and smart technologies have been implemented in ways that dynamically shift the power use of the homes. Collected by precise data logging technology, the total load of the homes can be determined. Though most of the loads are implemented through load and occupancy profiles, the elements of human interaction on humidity and latent heat in the homes are neglected for the most part. The absence of human presence and load alters the amount of stress that is exerted on the home. Daily activities such as cooking, cleaning, and exercising generate an environment that is currently not being modeled within the Pacific Northwest Nation Laboratory (PNNL) lab homes. The ability to use this technology will, "allow us to observe the occupancy of the home by operating it in unexpected ways" [2]. With the main goal of energy efficiency, this system will allow for a more realistic view of the typical home.

\subsection{Problems with Previous Technology}

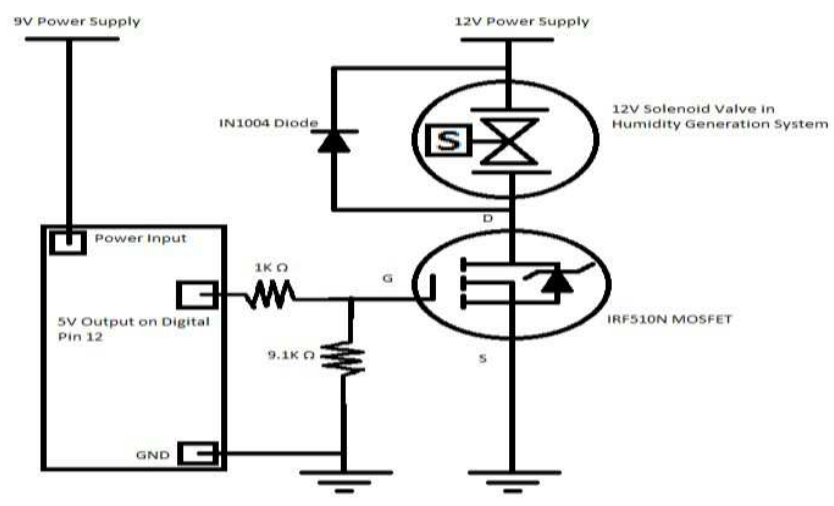

Figure 1. PNNL humidity generator [4]. 
PNNL has developed an occupancy simulation system to address this problem. At PNNL, Austin Winkelman developed standalone humidity generator. Below is the schematic detailing the specifics of the generator.

Controlled by an Arduino microcontroller, a solenoid is turned off and on based on the ASHRAE generation schedule. The following problems were encountered:

1) The hot plate needed to remain hot and on the entire time. This wasted energy and was a potential fire hazard.

2) Another issue was that the generator had no feedback system or $\mathrm{I} / \mathrm{O}$ interface. The amount of humidity generated and the frequency of generation was assumed to be correct.

3) The system had no way of differentiating from the latent and sensible heat loads of the environment.

4) Not expandable or portable. The location and the reservoir size remain static throughout the experiment.

\section{Ultra Sonic Controllable Humidifier}

With the idea of portability and flexibility in mind, a new humidity generation system has developed. It is able to differentiate between the sensible and latent heat loads, able to run for extended periods of time, and interface with the Campbell system to generate a feedback loop.

\subsection{Latent and Sensible Generation Profiles}

To facilitate research and promote consistent standards with analyzing residential homes, the Department of Energy has developed a "Building America Analysis Spreadsheets" [6]. These documents are specifically designed to provide a set of standard operating conditions. Specific building parameters are entered into the workbook. These include square footage, number of bedrooms, and predicated number of occupants. With this information, expected load profiles can be generated to reflect the input parameters. Specifically, this workbook is used to generate the latent and sensible electrical loads for new or existing homes.

From this workbook, an occupancy schedule was generated. This did not give numerical values for the total latent and sensible heat generated by occupants within the homes. This only gave the probability that the occupants will be in the home at specific hours of the day. The sensible and latent heats were calculated by PNNL engineers from the following equations.

$$
\frac{\text { Gal.H20 }}{\text { hour }}=\frac{T C}{\rho L}
$$

$\mathrm{T}=$ total latent heat

$\mathrm{C}=$ conversion from BTU to Watts

$\rho=$ density of $\mathrm{H} 20$

$\mathrm{L}=$ latent heat of evaporation

Latent Occupancy Load- reflected in heating water [6]

$$
\text { Latent Occupancy Load }=\frac{P N O}{C}
$$

$\mathrm{P}=$ probability of occupant within home

$\mathrm{N}=$ number of occupants

$\mathrm{O}=$ Occupant load per person per BTU per hour

$\mathrm{C}=$ conversion from $\mathrm{BTU}$ to Watts

\subsubsection{Humidity Generation System-Latent Load}

From the DOE building America house simulation protocol and latent heat calculations, it was determined that a total of $1.56 \mathrm{Gal} \mathrm{H} 20$ /day needed to be generated with a max generation hour of .085 Gal. This max hourly generation rate falls outside the max generation of the ultrasonic humidifier. To facilitate the total generation required, two human occupancy generators are installed within the PNNL lab homes, one in the kitchen and the other in the master bathroom. This promotes better penetration of the humidity throughout the house and will eliminate strain on a single unit.

The ultrasonic humidifier has been modified to generate both the latent and sensible heat. The transducer produces humidity that is independent of a heat source. This allows for complete controllability of both the latent and sensible heat to be measured and controlled. Through experiential measurements, the average generation rate of $4.55 \mathrm{ml}$ of $\mathrm{H} 2 \mathrm{O}$ vapor/min was found. This was generated through the experimental measurement of the evaporated water over a given 10 minute interval of time. The data collected can be seen below.

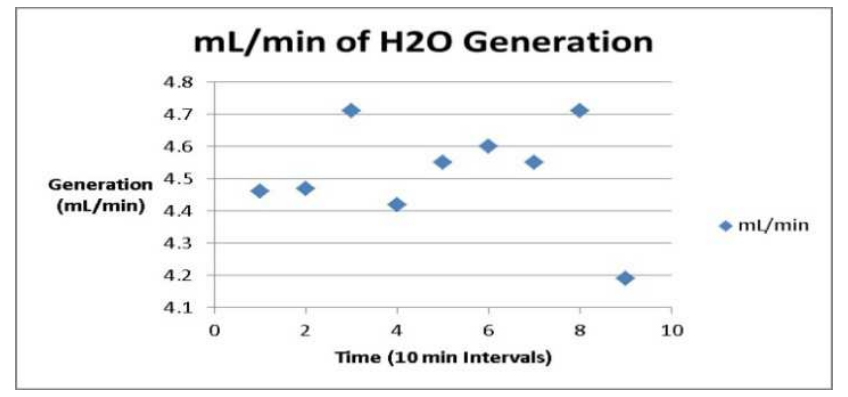

Figure 2. Experimentally measured latent heat load generation by the occupancy simulator.

\subsubsection{Humidity Generation System-Sensible Load}

Similar to the occupancy latent heat load generation, the sensible load was calculated by PNNL engineers through the use of Eq. 3. The sensible load will be generated through heating the water that is stored within the ultrasonic humidifier reservoir. From the given dynamics equations the final temperature of the water was found.

$$
Q=m c \Delta T
$$

$\mathrm{m}=$ mass in grams

$\mathrm{c}=$ specific heat of water

$\Delta \mathrm{T}=$ change in $\mathrm{H} 2 \mathrm{O}$ and ambient temperature

$\mathrm{Q}=$ Sensible heat in Joules

In this equation it is assumed that the transducer reservoir is always full. This has and expected weight of $64 \mathrm{~g}$ of $\mathrm{H} 2 \mathrm{O}$. Ambient air and water temperature are recorded and used to generate the change in temperature. The max sensible load 
seen within a 24 hour period is $193 \mathrm{~W}$. This needs to be generated over the course of 1 hour. From the above equation the water temp will need to be heated to $147.2 \mathrm{~F}$.

The heating of the water is done through a metal heating element that has been installed into the transducer reservoir. A feedback loop is generated between the temperature sensor within the reservoir and the heating element. It is attached with water tight gaskets to prevent water penetration into the control housing. The water will be constantly heated until it matches the required heat to compensate for the sensible heat load. Due to the limited size of the reservoir, an equilibrium temperature will penetrate through the full liquid without the required assistance of a mixing mechanism. Using a 3D printer, a housing for the heater was made to expose the maximum surface area, of the element, to the surrounding water. Below is a schematic of the housing.

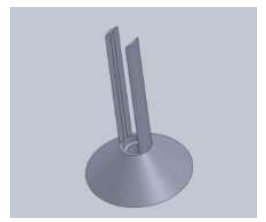

Figure 3. Heating element housing

\section{Control System}

The control systems were chosen based on previous engineering knowledge of the technology, a limited budget, and the ability to interface with the existing technology within the lab homes.

\subsection{Control System-Data Acquisition and Wireless Control}

A control system has been integrated into the existing enclosure of the humidifier. A 3D printer has been used to expand the enclosure of the humidifier to include all controlling peripherals. The main control system is the Arduino Uno rev3 micro controller. The controller uses the Atmel Atmega328 microprocessor and has a limited memory of $32 \mathrm{~Kb}$. The main peripheral mods that are installed are the relay and xbee shields. This allows for 4 relays to control via the Arduino. Above is a picture of the control system.

The 4 relays can be controlled by the $5 \mathrm{v}$ analog pins on the Arduino. The ultrasonic transducer and heating element are attached to individual relays. $120 \mathrm{~V}$ is supplied to the Arduino and allows for the switching to control the transducer and heating element. This is supplied by the "master" relay. The transducer and heating element remain on only as long as necessary to generate the required sensible and latent heat for the given hour.

The Arduino is equipped with an Xbee shield that will allow for wireless communication between the Arduino and existing Campbell data acquisition system. The Xbee has a large band width for wireless communication. The frequency of communication can be altered to not interfere with other wireless technology within the lab homes. Below is a schematic of the Xbee and Arduino interconnections.

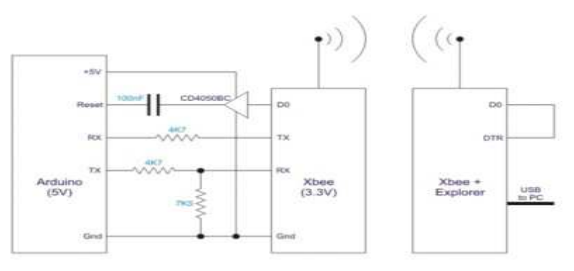

Figure 4. Xbee Arduino architecture schematic

The integrated Campbell scientific control system is the industry standard for data logging. Currently, this data logger is used within the lab homes to aggregate all of the sensor data. It is equipped with $12 \mathrm{v}$ and $5 \mathrm{v}$ channels that can power the Xbee. A transmitter Xbee is installed to interface between the Campbell and humidifier. The Campbell reads the internal relative humidity of the home or responds to a humidity schedule. If humidity is needed, the Campbell sends bits of information to the Xbee which will then transmit this data to the Arduino. Below is a picture of the described feedback loop.

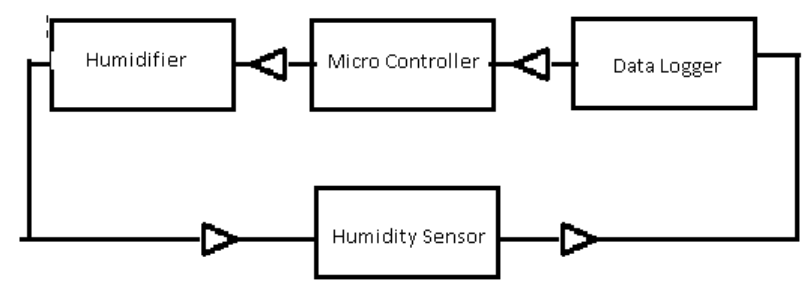

Figure 5. Control system feedback loop for the occupancy generator

\subsection{Water Control}

Based on the location of installation, a simple bypass or float system has been installed into the occupancy generator. Below is a simple drawing of the two methods used to control the water flow and reservoir water level.

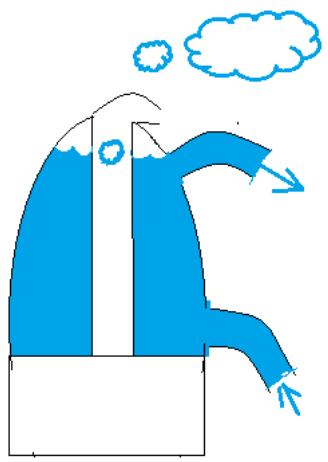

Method 1

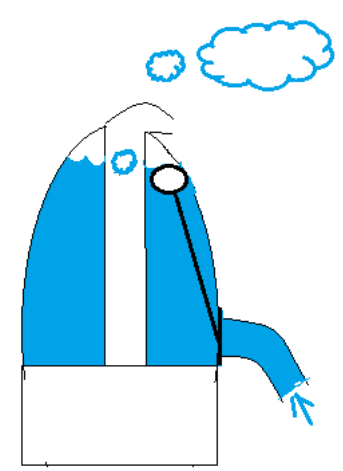

Method 2
Figure 6. Reservoir control and fill methods

Method 1: Within the kitchen, predefined water draws have been programmed to happen on the hour via the Campbell control system. Method 1 utilizes this almost constant stream of water to ensure that the water reservoir is constantly full. Due to the fact that the occupancy generator only produces 
1.56 gallons of $\mathrm{H} 2 \mathrm{O}$ per day, there is no possible way for the reservoir to run out of water within this scheme. Because the water is being bypassed, there is no possible way for the Reservoir to be over filled causing water damage in the lab. Method 2: This method has been implemented into the master bedroom. This area is not subject to water draws based on the Campbell's occupancy schedule. A simple float mechanism is installed into the second human occupancy generator. Installed within the master bathroom faucet, the reservoir will remain constantly full while preventing over flow.

\subsection{Control System-Safety mechanisms}

Both PNNL and Washington State University put a large emphasis on the safety culture with the laboratory settings. This has been continued within this project with the belief that a system product is useless if it is unsafe for the user and environment. Within the human occupancy generator, there are a few redundancies that promote safety.

1) Relay control- from experimental trial and error, the relay for the heating element should be in the on position no longer than an hour. If the relay remain on for greater than a predefined time this could cause damage to the transducer reservoir or become a fire hazard. As a safety control, the relay will periodically cycle off and on with a frequency of 1 time per 5 minutes. This ensures that the relay is working properly. If during this cycle, the relay remains closed for a period of greater than 10 minutes, the relay is assumed to have failed and the master relay will trip.

2) Temperature control - equipped with a temperature sensor, the control system monitors the temperature of the water that is within the reservoir. Knowing that the temperature should never surpass $147.2 \mathrm{~F}$, the arduino can trip the master relay if the water is heated above this set point for any sustained period of time for any reason.

3) Humidity Control - taking advantage of the installed humidity sensors within the lab homes aids in the protection feedback loop. The max humidity set point is known. If too much humidity is detected by the Campbell data logging technology, a signal will be sent wirelessly to the arduino. This will cause the arduino to trip the master relay.

4) Testing - It is recommended that the master relay be tested bi-monthly to ensure that it is fully functional.

The implementation of these 4 safeguards will ensure that the human occupancy generator is operating as expected and will not generate increased risk of fire or over humidification of the laboratory space.

\section{Benefits}

The benefits of the new occupancy generator are as follows:

1) The distribution of the sensible and latent heat loads. This allows for a more accurate representation and manipulation of an already dynamic system.

2) External control by data logging technologies.
3) Transducer eliminates the need for a hot plate.

4) Accurate and measureable load generation.

5) Portability due to expanded water reservoir.

Using this system, PNNL are able to achieve fairly accurate results for both the sensible and latent heat loads.

\subsection{Cost Estimation}

Table 1. Cost estimation for the project

\begin{tabular}{ll}
\hline Item & Cost $\mathbf{( \$ )}$ \\
\hline Arduino Uno & 24.51 \\
Relay Shield & 14.9 \\
Xbee & 19 \\
Humidifier & 29.99 \\
Heating Element & 10.99 \\
PSU & 6.99 \\
Total & 106.38 \\
\hline
\end{tabular}

Above is an aggregation of the total price of the components of the occupancy generator. This is only for one unit and does not reflect the total cost of the Campbell data logging technology. It can be seen that the price is substantially lower than many other technologies currently on the market that allow for expanded control of the laboratory environment. It would be difficulty to find a comparable ultrasonic humidifier that allows for any advanced user input for less than $\$ 150$. This product undercuts competing products on programmability, flexibility, and price point.

\section{Conclusion}

Adaptation of a ultrasonic humidifier with a built in control system has allowed for a more accurate means of controlling and measuring the dynamic environment generated in a residential or laboratory setting. Collaboration between PNNL and WSU has allowed for the further expansion of this topic into uncharted areas.

\section{References}

[1] ASHRAE (2008). “ASHRAE Standard 160P” Retrieved $12 / 15 / 20213$

[2] Fang, X. et al. (2011). "Field Test Protocol: Standard Internal Load Generation for Unoccupied Test Homes". NREL retrieved 12/17/2013

[3] Lee, Yoo. (2011). "Simulating Human Behavior and Its Impact on Energy Uses". University of Pennsylvania. Retrieved 12/15/2013.

[4] TenWolde, A. et al. (2007). "The Effect of Indoor Humidity on Water Vapor Release in Homes" ASHRAE. Retrieved $12 / 17 / 2013$

[5] Winkelman, A (2012) "Humidity Generation System" PNNL. Retrieved 12/15/2013.

[6] DOE, (2012). "Building America Workbook." Department of Energy. Retrieved 3/12/2014 